MATEC Web of Conferences 22,05007 (2015)

DOI: $10.1051 /$ matec conf/20152205007

(C) Owned by the authors, published by EDP Sciences, 2015

\title{
A Study on the Simulation of New Linear Transducer Based on the Ul- trafast Ultrasonic Imaging Technology
}

\author{
Ning Guo ${ }^{\mathrm{a}}$, Weiqiang Ye ${ }^{\mathrm{a}}$, Congzhi Wang* \& Hairong Zheng \\ Shenzhen Institutes of Advanced Technology, Chinese Academy of Sciences, Shenzhen, China
}

\begin{abstract}
The ultrafast ultrasonic imaging technology based on the transmitting/receiving principle of plane wave has become a research hotspot in the ultrasonic field of international medicine. It is hopeful that the technology replaces the traditional focus scanning ultrasonic imaging technology to be widely used clinically. This paper not only verifies the significant improving effect of coherent compound imaging algorithm on plane wave imaging resolution through the simulation of Field II software, but also proposes a new hypothesis that the Non-Elevation-Focused Probe (NEFP) is more helpful in further improving the imaging effect of plane wave imaging technology compared to the traditional Elevation-Focused Probe (EFP). In order to verify this hypothesis, this paper makes a comparative analysis on the plane wave imaging effect of the two kinds of transducers with the mode of emissive acoustic beam by controlling the elevation direction of probe (perpendicular to the short side direction of array element orientation) through parameters simulation. Results indicate that the contrast ratio of NEFP probe of near field imaging is significantly enhanced compared to that of EFP probe. Imaging resolutions of both probes are reduced when scattered elements deviate from the center of elevation direction. But the resolution of NEFP probe declines less.
\end{abstract}

Keywords: plane wave; ultrasonic probe; compound imaging; elevation focusing

\section{INTRODUCTION}

The traditional ultrasonic imaging produces a complete image by electronic scanning through multiple focus emission ${ }^{[1]}$, so the frame frequency of imaging is relatively low. With the emergence of imaging technologies like cerebral function imaging and real-time three-dimensional ultrasound imaging, requirements on the frame frequency of imaging are becoming higher and higher. Traditional frame frequency speed is now difficult to satisfy new imaging

modes. Plane wave emission is a method that can improve the frame frequency of imaging rapidly ${ }^{[2]}$. It is able to cover the entire imaging area with one emission, largely increasing the frame frequency of imaging. In order to solve the problem of poor signal to noise ratio, contrast ratio and resolution of plane wave imaging, the method of coherent compound imaging method of plane wave is put forward by French scholars ${ }^{[3]}$.

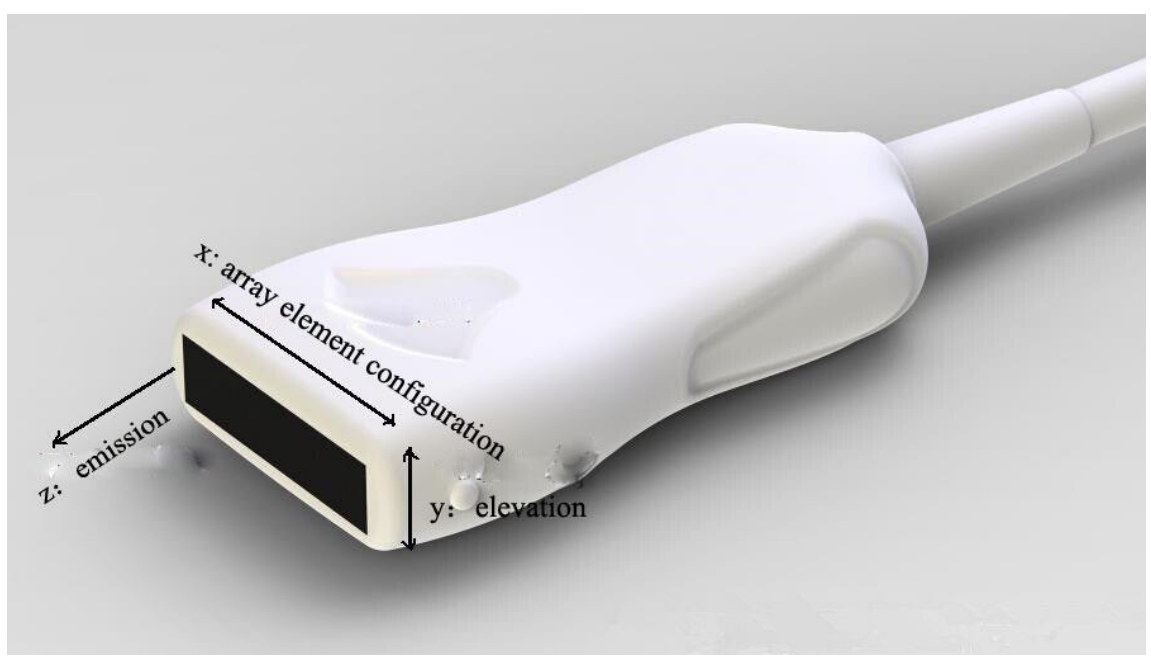

Figure 1. Diagram of ultrasonic probe

* Corresponding author: cz.wang $@$,siat.ac.cn; ${ }^{\text {a }}$ they made the same contribution to the article

This is an Open Access article distributed under the terms of the Creative Commons Attribution License 4.0, which permits unrestricted use, distribution, and reproduction in any medium, provided the original work is properly cited. 
The coherent compound imaging is an ultrasonic imaging technology that sends sound waves in different directions and fuses received echo data in certain rules. It is able to reduce speckle noises and artifacts so as to improve image definition, contrast and resolution. However, multiple times of emissions are needed and the frame frequency is reduced, so the time of emission of coherent compound imaging should be ration$\mathrm{al}^{[4]}$.

Ultrasonic transducer probe has the function of ultrasonic wave emission and receiving, the structure of which has great influence on imaging effect ${ }^{[5]}$. In terms of longitudinal beam control of ultrasonic transducer, the current beam control methods of linear array mainly include electronic control adjustment and acoustic lens adjustment ${ }^{[6]}$. Electronic adjustment technology achieves the desired beam effect by controlling the time delay of each array element of the transducer. Acoustic lens adjustment changes the shape of beam by altering radians of the matching layer and acoustic lens. In a normal ultrasonic linear array transducer, there is no incision along the short edge direction of chip (namely the elevation direction of probe) and an elevation focus is generated in the elevation direction of probe by changing radians of the matching layer and acoustic lens. The multi-dimensional imaging that attracts much attention in recent years ${ }^{[7,8]}$ incises the chip into several pieces along the short edge direction. The acoustic beam control technology adds electronic delay to separated chips in two-dimensional direction so as to achieve the desired acoustic beam focusing effect.

At present, researches on plane wave imaging mainly focus on imaging methods. However, researches on the relationship between the structure and working method of ultrasonic probe and plane wave imaging effect are neglected. All the researches on plane wave imaging adopt the traditional linear array ultrasonic probe, the diagram of which is shown in Figure 1. Non-delay emission is adopted horizontally (width direction of probe) to form plane wave. But there is a physical focus formed by acoustic lens in the elevation direction of probe, which is not a real and pure plane wave. A new hypothesis is put forward according to this problem that it might help to improve the imaging effect of the plane wave imaging technology by adopting the Non-Elevation-Focused Probe (NEFP) instead of the traditional Elevation-Focused Probe (EFP). In order to verify this hypothesis, this paper first implements the coherent compounding imaging algorithm of plane wave by the simulation of Field II software and compares the difference between the effect of multi-angle coherent compound imaging and the effect of single angle plane wave imaging. It then controls the emissive beam mode of the elevation direction of probe by altering simulation parameters and makes a systematic and comparative analysis on plane wave imaging effects of two transducers. The result suggests that the plane wave imaging effect of NEFP probe is superior to that of EFP probe in certain aspects.

\section{COMPOUND IMAGING ALGORITHM OF PLANE WAVE}

The algorithm mostly used in ultrasonic imaging is the delay superposition algorithm ${ }^{[9]}$. It is widely used in practical measurement due to its simplicity and fast arithmetic speed. This algorithm is adopted for imaging in the generation of a single picture of plane wave. Major steps of the algorithm are provided below.

DAS imaging is in the way of direct superposition after the time delay of received signals, which is shown in the following formula:

$$
Y(t)=\sum_{m=1}^{M} \sum_{n=1}^{N} w_{m, n} x_{m, n}\left(t-\tau_{k}\right)
$$

Here, ${ }^{\tau} k$ is the time delay of focus $k, M$ is the number of emitted array elements, and $N$ is the number of received array elements. In practical imaging, received echo signals are formed into a beam after $\mathrm{AD}$ sampling. So, the discrete mathematical model can be established as follows. As for the focus $k$, suppose:

$$
x_{m, n}(k)=x_{m, n}\left(t-\tau_{k}\right)
$$

As for the focus $k,{ }^{x_{m, n}}{ }^{(k)}$ stands for the signal of the $\mathrm{m}^{\text {th }}$ array element emission and the $\mathrm{n}^{\text {th }}$ array element receiving after time delay. So, $X_{m}(k)$ stands for the signal received by the full array after the $\mathrm{m}^{\text {th }}$ array element emission, which can be expressed as:

$X_{m}(k)=\left[x_{m, 1}(k), x_{m, 2}(k), \cdots, x_{m, n}(k), \cdots, x_{m, N}(k)\right]$

A discrete mathematical model can be obtained from Formula (1) and (2):

$$
\begin{aligned}
& Y(k)=\sum_{m=1}^{M} W_{m}(k) X_{m}(k)=\sum_{m=1}^{M}\left[w_{m, 1}, w_{m, 2}, \cdots w_{m, N}\right] \bullet \\
& {\left[x_{m, 1}(k), x_{m, 2}(k), \cdots, x_{m, n}(k), \cdots, x_{m, N}(k)\right]}
\end{aligned}
$$

Here, $w_{m}(k)=\left[w_{m, 1}, w_{m, 2} \cdots w_{m, N}\right]$ is a weighted value, standing for the weighted value of the received signal of focus $k$ when the $\mathrm{m}^{\text {th }}$ array element is emitted. Set the weighted value $W_{m}(k)$ as a fixed value, such as rectangular window, Hanning window and Blackman window. The function weighting of rectangular window is adopted in this paper.

Stolt transport theory is frequently used in seismic imaging detection, featuring fast speed of implementation. But this algorithm is not suitable for situations with fast wave velocity changes. Its application is limited in some ways due to the significant changes of propagation velocity of seismic wave in stratum ${ }^{[10]}$ The propagation velocity of ultrasound approximates a fixed value, namely $1540 \mathrm{~m} / \mathrm{s}$. The defect of large variation amplitude of propagation velocity can be perfectly overcome by applying the algorithm in the medical field of fast ultrasonic imaging so as to realize 
ultrasonic imaging of high frame frequency. Refer to Literature ${ }^{[11]}$ for detailed steps of the algorithm. Positions of scattered elements can be solved according to Stolt transport theory. The emissive angle of compound imaging is known, so the offset can be solved from the geometrical relationship. Compound and superpose in accordance with the offset ${ }^{[3]}$ so as to realize the composite superposition of images.

\section{SIMULATION EXPERIMENT AND IMAGING RESULTS}

\subsection{Coherent compound imaging simulation}

In order to verify the imaging effect of coherent compound imaging algorithm, this paper first conducts a simulation of the coherent compound imaging method, then compares imaging effects of two transducer models through the simulation, and finally makes an analysis on simulation data.

Firstly, use MATLAB software to carry out the simulation experiment through the ultrasonic simulation program of Field II by setting transducer parameters. Suppose the sound propagation medium is human tissue, the sound velocity is set as $1540 \mathrm{~m} / \mathrm{s}$ with no regard to sound attenuation and the heterogeneity of tissue. The emission frequency of transducer is $7.5 \mathrm{MHZ}$, the number of array elements is 128 , the interval between array elements is $2.7 \mathrm{~mm}$, and the length of elevation direction is $5 \mathrm{~mm}$. In order to achieve the focus effect of acoustic lens in elevation direction through simulation, array elements are separated into 10 portions so as to realize electron focusing. The elevation focus depth is $30 \mathrm{~mm}$. In the simulation experiment, 30 point scattering objectives are verified. The depth of simulation imaging is $0-80 \mathrm{~mm}$ and the 30 points are distributed regularly between 11 and $65 \mathrm{~mm}$. The interval of points in depth direction is $6 \mathrm{~mm}$. The horizontal position locates $-5 \mathrm{~mm}$ and $10 \mathrm{~mm}$ and the lengthwise position locates the central plane of $0 \mathrm{~mm}$. The simulation is marked as simulation 1. The simulation experiment is carried out through the compound imaging algorithm. The compound angle changes with an interval of $\Delta \theta=\mathrm{pi} / 180$ from $\theta=$ $-((\mathrm{N}-1) / 2)^{*} \mathrm{pi} / 180$. N stands for the number of compound imaging. Results of compound imaging are shown in Figure 2. In order to further analyze the resolution and contrast ratio of different methods, cross sections of different depths in Figure 2 are selected for analysis. Lateral sections of different amplitudes at $30 \mathrm{~mm}$ and $60 \mathrm{~mm}$ are shown in Figure 3, where a stands for that of $30 \mathrm{~mm}$ and $\mathrm{b}$ stands for that of $60 \mathrm{~mm}$. The comparison of two indicators, the Full Width at Half Maximum (FWHM) and the Peak Side-lobe Level (PSL) at $60 \mathrm{~mm}$, is provided in Table 1. FWHM refers to the waveform width at the half peak of the main lobe. The smaller FWHM is, the better the image resolution is. PSL is the ratio of the main lobe peak strength and side-lobe peak strength.
The smaller PSL is, the better side-lobe is inhibited and the better the image contrast ratio is. There are three peaks in the figure. Take the average of parameters required by the three peaks.

In Figure 2, (a), (b) and (c) respectively stands for the coherent compound imaging result of 1,9 and 21 images. It can be seen that there are more near-field artifacts in the imaging of one image. Near-field artifact is mainly caused by side-lobes of ultrasonic emission field. The resolution and the contrast ratio of the coherent compound imaging of 21 images are the best and the near-field artifacts are perfectly inhibited as well. The resolution and the contrast ratio of the coherent compound imaging of 9 images are superior to those of the plane wave imaging of a single image. But the compound imaging effect of 9 images is not as good as that of 21 images. Lateral transformation curves of 1,9 and 21 compound images at $30 \mathrm{~mm}$ are provided in Figure 3.a and lateral transformation curves at $60 \mathrm{~mm}$ are provided in Figure 3.b. It can be clearly seen from Figure 2 that the full width at half maximum of the compound image of 21 images is the narrowest and the side-lobe is perfectly inhibited. And resolution and contrast ratio are the best as well. The compound image of 9 images is in the second place and the emission and receiving of one image come last. Detailed parameters of compound imaging of different images are listed in Table 1 after the quantitative analysis on data in Figure 3. It can be seen from data in Table 1 that the value of FWHM decreases and the imaging resolution increases with the increase of compound images while the value of PSL decreases with the increase of imaging contrast ratio. It can be also seen that the resolution of near field is superior to

Table 1. Parameter comparison of different numbers of compound imaging

\begin{tabular}{|l|l|l|l|l|}
\hline $\begin{array}{l}\text { Numbers } \\
\text { of com- } \\
\text { pound } \\
\text { imaging }\end{array}$ & \multicolumn{2}{|l|}{ FWHM/mm } & \multicolumn{2}{l|}{ PSL/dB } \\
\cline { 2 - 5 } & $30 \mathrm{~mm}$ & $60 \mathrm{~mm}$ & $30 \mathrm{~mm}$ & $60 \mathrm{~mm}$ \\
\hline 1 & 2.125 & 2.625 & -80 & -100 \\
\hline 9 & 1.706 & 1.837 & -110 & -140 \\
\hline 21 & 1.049 & 1.112 & -130 & -145 \\
\hline
\end{tabular}

that of distant field while the contrast ratio of distant field is superior to that of near field.

\subsection{Simulation comparison of different types of probes}

In order to verify the imaging effect of EFP probe and NEFP probe, this paper adopts Field II to carry out a simulation experiment. Simulation parameters are mentioned in the earlier section. 
MATEC Web of Conferences

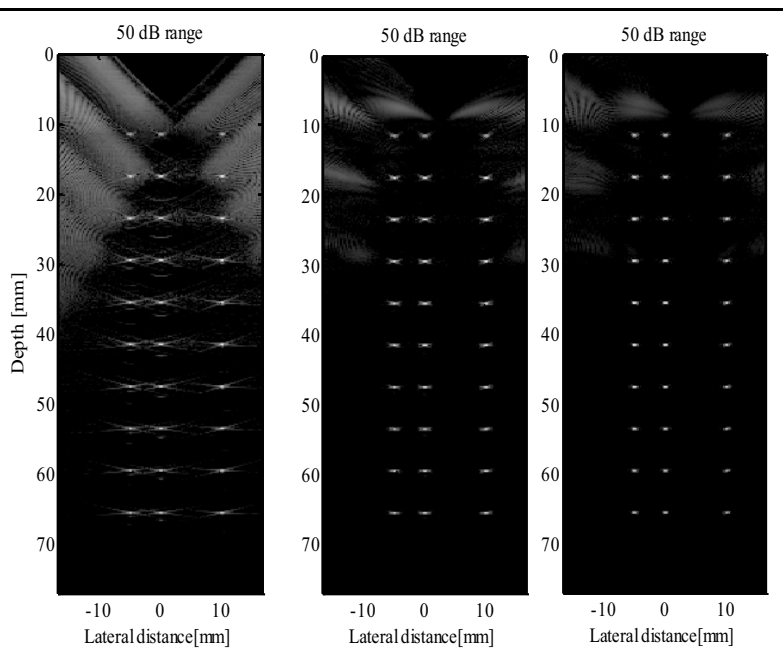

Figure 2. Imaging results of scattering points of compound imaging

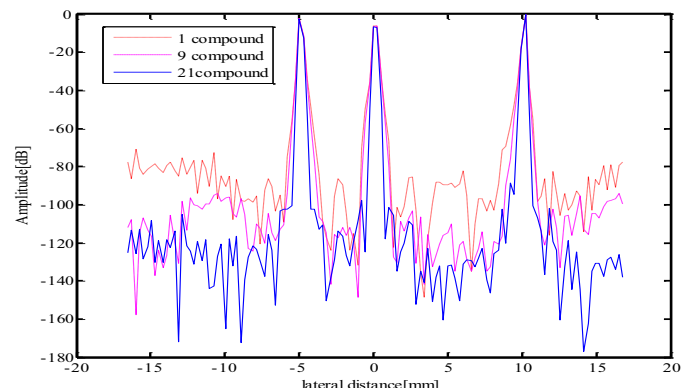

a

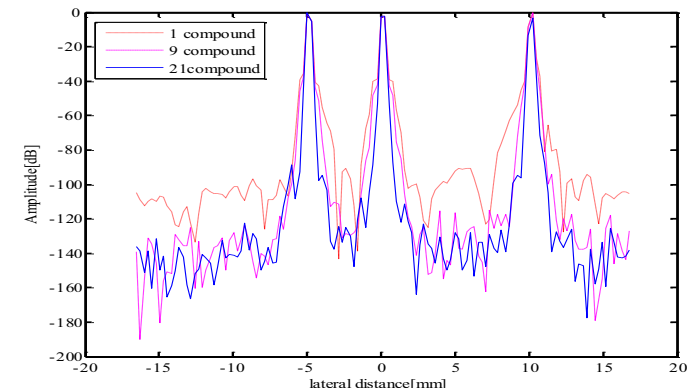

$\mathrm{b}$

Figure 3. Lateral transformation curves at different depths

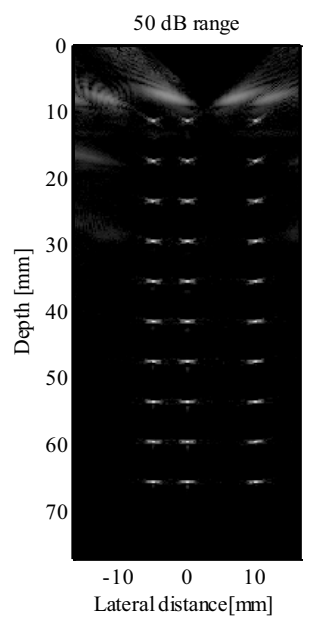

a. Imaging of NEFP
$50 \mathrm{~dB}$ range

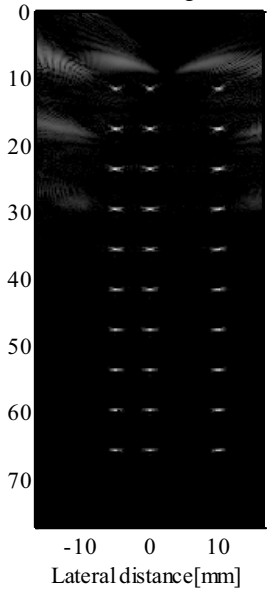

b. Imaging of EFP

Figure 4. Imaging results of simulation 1 in different probe mode 


\section{ICETA 2015}

The elevation focusing depth is set as $30 \mathrm{~mm}$ through electron focusing. NEFP probe and EFP probe are separated into 10 portions equally in the elevation direction of array element without electron focusing. Other parameters of the two probes are the same. There is not only the imaging of simulation 1 but also the imaging of simulation 2 in the observation of compound imaging effect of two probes of 9 images. All scattering points of simulation 2 deviate from the central plane by $1.5 \mathrm{~mm}$ compared to those of simulation 1. Other parameters remain the same. The imaging effect of simulation 1 is shown in Figure 4 and the imaging effect of simulation 2 is shown in Figure 7. In order to make a better comparison of imaging effects of different focus probes, data of cross sections at

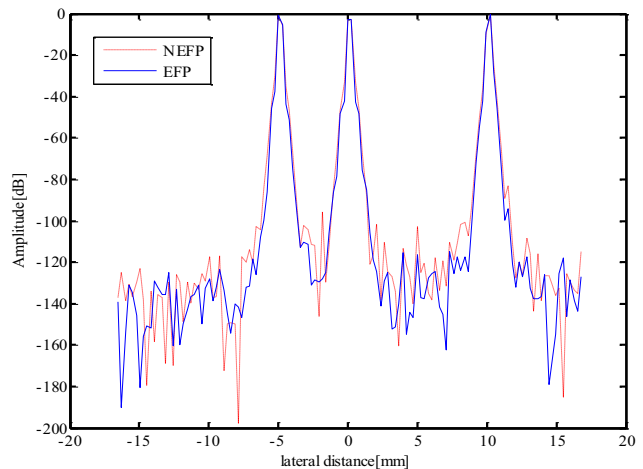

a. Data at the depth of $60 \mathrm{~mm}$

Figure 5. Imaging transformation curves of different probes

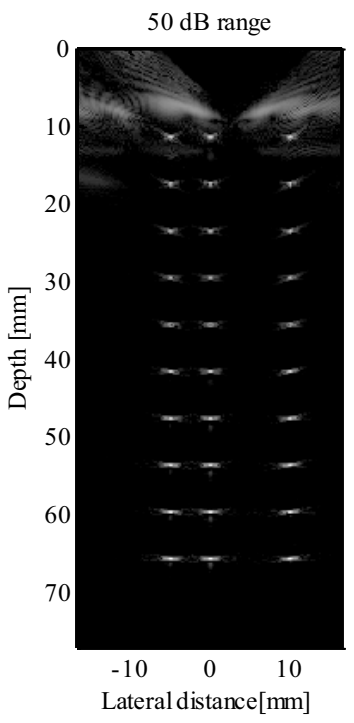

a. Imaging of EFP
$30 \mathrm{~mm}$ and $60 \mathrm{~mm}$ are analyzed from various aspects.

In Figure 4, $a$ and $b$ stand for the imaging of simulation 1 of different probes. It can be seen from the comparison between $\mathrm{a}$ and $\mathrm{b}$ that the imaging in each depth in figure $b$ is more even and the imaging resolution in distant field is higher. In Figure 5, a and b respectively stand for the analysis on different probes at the depth of $60 \mathrm{~mm}$ and $30 \mathrm{~mm}$. It is observed that the difference between EFP probe and NEFP probe in influencing imaging resolution and contrast ratio of scattered elements is not obvious at the depth of $60 \mathrm{~mm}$ and the difference in resolution of the two probes is not obvious as well at the depth of $30 \mathrm{~mm}$. However, the contrast ratio of NEFP probe is evidently superior to that of EFP probe.

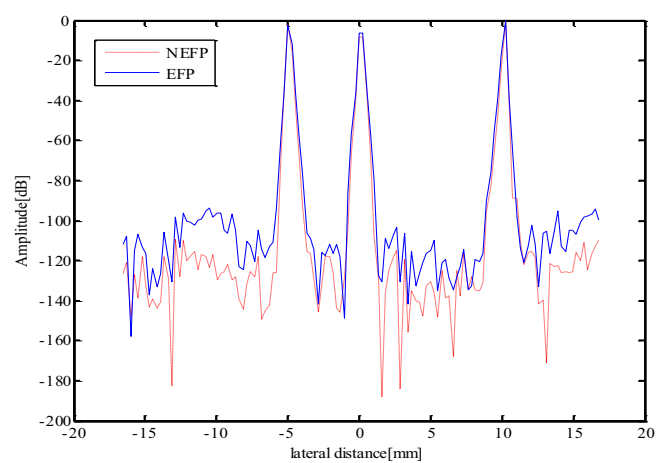

b. Data at the depth of $30 \mathrm{~mm}$

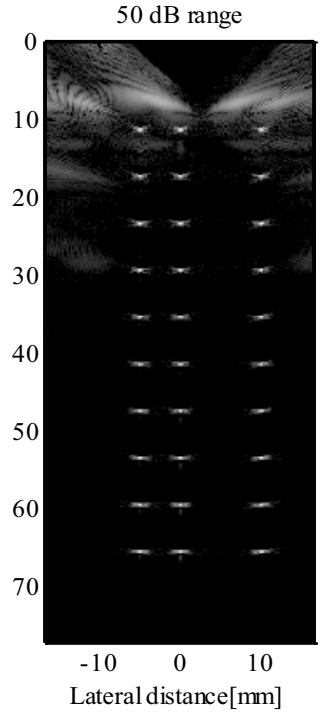

b. Imaging of NEFP

Figure 6. Imaging results of different probe modes in simulation 2 


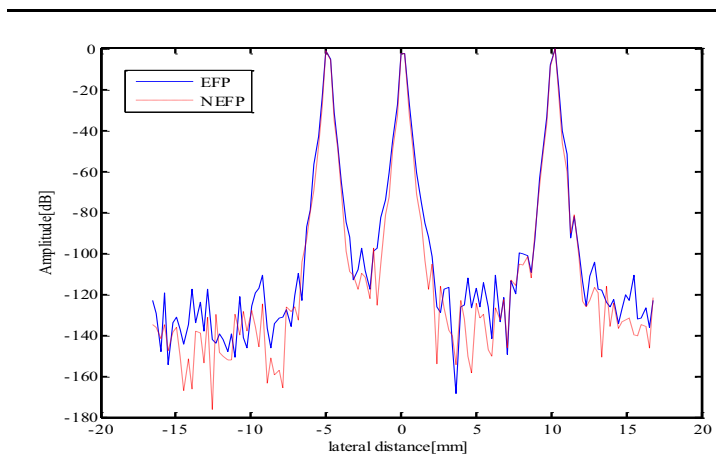

a. Data at the depth of $60 \mathrm{~mm}$

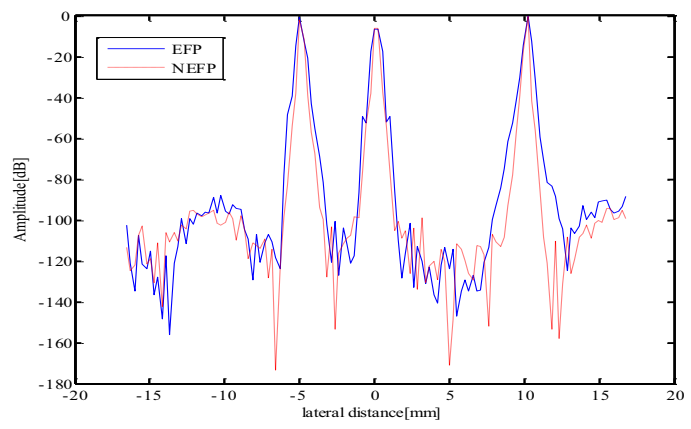

b. Data at the depth of $30 \mathrm{~mm}$

Figure 7. Imaging transformation curves of different probes

Table 2. Comparative analysis of two probes in different imaging ways

\begin{tabular}{|l|l|l|l|l|l|l|l|l|}
\hline Parameters of probe $/ \mathrm{mm}$ & \multicolumn{2}{l|}{ Simulation 1} & \multicolumn{3}{l|}{ Simulation 2} \\
\cline { 2 - 10 } & $\begin{array}{l}\text { FWHM } \\
30 \mathrm{~mm}\end{array}$ & $\begin{array}{l}\text { FWHM } \\
60 \mathrm{~mm}\end{array}$ & $\begin{array}{l}\text { PSL } \\
30 \mathrm{~mm}\end{array}$ & $\begin{array}{l}\text { PSL } \\
60 \mathrm{~mm}\end{array}$ & $\begin{array}{l}\text { FWHM } \\
30 \mathrm{~mm}\end{array}$ & $\begin{array}{l}\text { FWHM } \\
60 \mathrm{~mm}\end{array}$ & $\begin{array}{l}\text { PSL } \\
30 \mathrm{~mm}\end{array}$ & $\begin{array}{l}\text { PSL } \\
60 \mathrm{~mm}\end{array}$ \\
\hline NEFP & 1.771 & 2.188 & -130 & -135 & 1.875 & 2.187 & -120 & -150 \\
\hline EFP & 1.458 & 1.875 & -105 & -135 & 2.500 & 2.292 & -120 & -135 \\
\hline
\end{tabular}

In Figure 6, a and b stand for the imaging of simulation 2 of different probes. Differences between the two images cannot be seen clearly from the comparison of figure $a$ and figure $b$. For better comparison, figure 7.a stands for the data comparison of two probes at the depth of $60 \mathrm{~mm}$ and figure $7 . \mathrm{b}$ stands for the data comparison of two probes at the depth of $30 \mathrm{~mm}$. It can be concluded that the influence of EFP probe and NEFP probe in imaging resolution and contrast ratio of scattered elements is not obvious at the depth of $60 \mathrm{~mm}$ and the difference in contrast ratio of the two probes is not obvious as well at the depth of $30 \mathrm{~mm}$. However, the resolution of NEFP probe is obviously superior to that of EFP probe.

The quantitative and comparative analysis on parameters of different probes is conducted for further analysis and comparison. Table 2 is given below. It can be clearly seen from data in Table 2 that the imaging resolution of NEFP probe in near field is lower than that of EFP probe in simulation 1 while the contrast ratio is higher. The resolution of NEFP probe in near field is clearly higher than that of EFP probe in simulation 2 while the difference of contrast ratio is not obvious. In distant field, resolutions and contrast ratios of probes in different simulations are not obvious and the difference in imaging effect of probes is relatively small as well.

\section{CONCLUSION}

Poor image resolution and contrast ratio will be obtained if traditional beam forming algorithm is applied in plane wave imaging. So, it is not suitable for ultrafast ultrasonic imaging technology. This paper realizes fast imaging of plane wave through the method of coherent compound imaging. Simulation results indicate that higher imaging resolution can be achieved by this method. This paper makes a further comparison between NEFP probe and EFP probe. It can be found from the simulation experiment that the contrast ratio of NEFP probe in near field is obviously superior to that of EFP probe. It can be also found from the analysis on the same probe at different depths that the resolution of NEFP probe is slightly low in distant field and the contrast ratio of EFP probe is relatively large in distant field. But the change of resolution is not obvious. Through the comparison of two probes' imaging at the central position and the non-central position, it can be seen that imaging qualities of two probes at the deviated central position are relatively reduced. The resolution of NEFP probe and the contrast ratio of EFP probe are reduced. It can be concluded from the data comparison that NEFP probe is indeed superior to that of EFP probe in certain aspects despite merits and defects of both probes in plane wave imaging effect. It provides a new research idea for the further improvement of ultrasonic plane wave imaging effect and the clinical application. It is also of great significance for the development of medical ultrasonic imaging.

\section{REFERENCES}

[1] M. Karaman, P.C. Li. \& Matthew O’Donnell. 1995 Synthetic aperture imaging for small scale systems, IEEE Transactions on Ultrasonic, Ferroelectrics, and Frequency Control, 42: 429-442. 
[2] Shen, M. F., Zhang, Q. \& Li, D. L., et al. 2011. Beam space sparse representation weighting beam forming for plane wave emission ultrasound instrument, IEEE Instrumentation and Measurement Technology Conference. Binjiang: 1-5.

[3] Montaldo G1, Tanter M, Bercoff J, Benech N. \& Fink M. 2009. Coherent plane-wave compounding for very high frame rate ultrasonography and transient elastography, IEEE Transactions on Ultrasonic, Ferroelectrics, and Frequency Control, 56(3): 489-506. doi: 10.1109/TUFFC.2009.1067.

[4] Forsberg F. 2004. Ultrasonic biomedical technology, marketing versus clinical reality. Ultrasonics, 42: 17-27.

[5] Li, Y. \& Li, M.D. 2005. Structure and features of ultrasonic array probe, Nondestructive Inspection, 1671-4423(2005) 06-01-05.

[6] Bai, P.R. \& Zhang, L.L. 2009. Simulation research on beam control technology of two-dimensional ultrasonic array transducer, Natural Science, 28(5).

[7] Foster, F S. 2000. Transducer materials and probe construction, Ultrasound in Medicine and Biology, 26(s1): s2-s5.

[8] Fronheiser, M P. \& Light E D, et a1. 2006. Real-time 3D ultrasound with multiple transducer arrays, IEEE Transactions on Ultrasonic, Ferroelectrics, and Frequency Control, 53(1): 100-105.

[9] Peng, H. Introduction of Ultrasonic Imaging Algorithm (Second edition), Hefei: Press of University of Science and Technology of China, pp: 114-118.

[10]Egil S.Eide, 2000. Radar Imaging of Small Objects Closely below the Earth Surface, Department of Telecommunications, Norwegian University.

[11]G. F. Margrave. 2003. Elementary migration methods in numerical methods of exploration seismology, CREWES Educational Resources, Ed., pp.112-188. [OL]. Available:http://www.crewes.org/researchlinks/Freesoftware/n ummeth.pdf

[12]D. Garcia, L. L. Tarnec. 2013. Stolt's f-k migration for plane wave ultrasound imaging, IEEE Transactions on Ultrasonic, Ferroelectrics, and Frequency Control, 60(9).

[13] Jensen J. 1996. A. Field: A program for simulating ultrasound systems, Med. Biol. Engineering and Computing, 34(S1): 351-353. 